\title{
Predicting solute descriptors for organic chemicals by a deep neural network (DNN) using basic chemical structures and a surrogate metric
}

\author{
Kai Zhang, Huichun Zhang* \\ Department of Civil and Environmental Engineering, Case Western Reserve University, Cleveland, Ohio \\ 44106, United States \\ * Corresponding author email: hjz13@case.edu
}

The supporting information (a total of 28 pages including the cover sheet) contains:

Texts S1 - S6

Figures S1 - S14

Tables S1 - S3

Text S1. Model development/evaluation and comparison with other models

Text S2. Three dimension-reduction methods

Text S3. Chemical datasets and Gaussian process regression (GPR) modeling

Text S4. The surrogate metric based on $\log \mathrm{P}$

Text S5. Post hoc interpretation of the obtained PaDEL-DNN models

Text S6. Estimating the LFER descriptors for persistent, bio-accumulative, and toxic (PBT) chemicals

Figure S1. The distribution histogram for $E, S, A$, and $B$ in the pp-LFER dataset

Figure S2. Modeling performance during cross-validation for different algorithms using the PaDEL chemical representation. SVM-1 to -3 represent SVM with different kernel functions. Tree1 to -4 indicate models using different tree-based algorithms.

Figure S3. Predicted vs. experimental values for E, S, A, and B by the PaDEL-DNN models. Note, there are more than 1900 data points in each plot.

Figure S4. Comparison of the predictions for the pp-LFER descriptors by the PaDEL-DNN models (this research) and by the MLFER (the PaDEL package).

Figure S5. Five chemicals were randomly selected from the 305, 51, and 22 chemical sets, respectively. The chemicals in the first row belong to the 305 -chemical set minus the 51 chemicals; the second row showed 5 chemicals from the 51 -chemical dataset; the last row showed 5 chemicals from the 22-chemical set. 
Figure S6. The distribution of the prediction errors of $\log P$ for the chemicals from the $\log K_{\text {storage- }}$ lipid/water dataset.

Figure S7. Demonstration for applying the surrogate metric.

Figure S8. Structural and pp-LFER descriptors for cyclohexanamine.

Figure S9. Five nearest neighbors for cyclohexanamine based on the outputs of the last hidden layer of the PaDEL-DNN models.

Figure S10. Five nearest neighbors for cyclohexanamine based on the PaDEL representation.

Figure S11. Five nearest neighbors for cyclohexanamine based on the Tanimoto similarity.

Figure S12. Comparison of the model performance of the KNN models using the PaDEL chemical representation with (PaDEL_Re) and without (PaDEL) dimension reduction.

Figure S13. Comparison of the model performance on the full test dataset (PaDEL) and on two smaller test datasets (PaDEL-1H and PaDEL-2H).

Figure S14. Snapshot of the demo prediction for benzene by the offline web predictor.

Table S1. Summary of the datasets included in this research

Table S2. Comparison of the performance of MF-DNN using molecule fingerprints with different lengths

Table S3. Features included in the RDKit and PaDEL molecular representations used in this research 


\section{Text S1. Model development/evaluation and comparison with other models}

S1.1 Chemical representation generation: 1) The PaDEL package can provide constitutional, topological, electrostatic, geometric, steric, combination, hydrophobic, and basic quantum chemical information about chemicals (More detailed features were included in Table S3). RDkit can provide less diverse information, including constitutional, topological, electrostatic, geometric, hydrophobic aspects about chemicals. As for the molecular fingerprint, it can mostly provide constitutional information about chemicals.

2) To derive the three chemical representations, the SMILES of chemicals will be needed. For molecular fingerprints, multiple tools can be used to generate fingerprints such as the "RDKit" package and the "PaDEL" package. The methods for calculating the RDKit chemical representation are included in three modules from the "RDKit" package, ${ }^{1}$ namely "rdkit.Chem.rdMolDescriptors", "rdkit.Chem.Descriptors", and "rdkit.Chem.Descriptors3D". One can call these functions to calculate the chemical representation in a Python environment using SMILES as the input.

For the PaDEL chemical representation, a Java environment will be needed to run the stand-alone “PaDEL" package; ${ }^{2}$ however, a wrapped PaDEL (PaDELPy, https://github.com/ecrl/padelpy) was recently developed for running "PaDEL" from a Python environment.

3) The molecular fingerprint essentially only includes constitutional information of chemicals, such as functional groups and bonds. The RDkit representation includes much more information than simple constitutional one. The PaDEL representation further includes more information than the RDkit representation, in terms of both diversity and quantity for each type of chemical information. The more comprehensive information from the PaDEL representation naturally can better describe chemicals and, thus, achieve better predicting performance than the other two chemical representations. The dimension reduction results in the main text also supported such a conclusion. After performing dimension reduction on the PaDEL chemical representation, the input of 153 features only achieved slightly worse modeling performance than the models using all the features from the PaDEL representation, which suggests that these 153 features were critical for predicting the solute descriptors. However, only part of these critical features was included in the RDKit chemical representation, and even less was represented by the molecular fingerprint.

S1.2 Algorithm selection: Before the formal modeling, we compared several commonly used machine learning algorithms, including support vector machines (SVM) and some tree-based algorithms. As shown in Fig. S2, almost all the tested algorithms showed higher RMSEs than the DNN on the same training datasets. Meanwhile, SVM-3 which uses a "polynomial" kernel function 
showed RMSEs close to the DNN but had much higher variances in the RMSEs for each solute descriptor. In contrast, the DNN models not only had the lowest RMSEs but also very small variances in the RMSE values. Considering both the average RMSEs and the associated variances, DNN was used for the modeling in this study.

S1.3 Model development and evaluation: During the model development, Tensorflow ${ }^{3}$ was used to build DNN models and Hyperopt ${ }^{4}$ was used to optimize the hyperparameters, including the number of neurons in hidden layers, number of hidden layers, activation function, learning rate, and dropout rates. The optimum hyperparameters were selected based on the 5-fold cross-validation root-mean-square errors (RMSEs) of the training dataset. During the cross-validation, the training dataset was equally partitioned into 5 sub-datasets. One of the sub-datasets was used as the validation dataset, and the remaining 4 were used as the training dataset. This process was then repeated until each of the 5 sub-datasets was used once as the validation dataset. The RMSEs and $\mathrm{R}^{2}$ values were then calculated based on the cross-validation results for optimum hyperparameters selection. The best models were then evaluated on the test dataset. The above modeling process was repeated multiple times for every chemical representation to avoid possible biased data splitting and unreliable modeling results in one-time splitting.

S1.4 Comparison with QC-MLR: In this study, the RMSEs (or MSE) and the associated standard deviation were provided. So, the central limit theorem (Eq. S1) ${ }^{5}$ was used to compare the performance of the PaDEL-DNN models with that of reported models.

$$
R M S E_{1}-R M S E_{2} \sim N\left(0, \frac{s_{1}^{2}}{N_{1}}+\frac{s_{2}^{2}}{N_{2}}\right)
$$

where $s_{1}$ is the sample standard deviation of the first group of RMSE, $N_{1}$ is the sample size (number of chemicals) of the first group, and so forth. The one-sided p-value can be calculated by:

$$
p_{\text {value }}=1-\Phi\left(Z \leq \frac{R M S E_{1}-R M S E_{2}}{\sqrt{\frac{s_{1}^{2}}{N_{1}}+\frac{s_{2}^{2}}{N_{2}}}}\right)
$$

When comparing the prediction errors in terms of RMSEs for the "E" term by the PaDEL-DNN models $(0.1 \pm 0.01, \mathrm{~N}=1978)$ versus by the $\mathrm{QC}-\mathrm{MLR}^{6}(\mathrm{RMSE}=0.17, \mathrm{~N}=849)$, we can assume that the QC-MLR and the PaDEL-DNN models have similar standard deviations:

$$
H_{0}: R M S E_{1}-R M S E_{2}=0
$$




$$
\begin{gathered}
R M S E_{1}-R M S E_{2} \sim N\left(0, \frac{0.01 \cdot 0.01}{1978}+\frac{0.01 \cdot 0.01}{849}\right)=N\left(0,1.68 \times 10^{-7}\right) \\
p_{\text {value }}=1-\Phi\left(Z \leq \frac{0.17-0.1}{\sqrt{1.68 \times 10^{-7}}}\right)<0.01
\end{gathered}
$$

So, we may reject $H_{0}$ at $\alpha=0.01$.

Similarly for the "B" terms, where the RMSE by PaDEL-DNN was $0.1 \pm 0.01$ and the RMSE by the QC-MLR was $0.12,{ }^{6}$ we can repeat the calculations above:

$$
\begin{gathered}
R M S E_{1}-R M S E_{2} \sim N\left(0, \frac{0.01 \cdot 0.01}{1978}+\frac{0.01 \cdot 0.01}{849}\right)=N\left(0,1.68 \times 10^{-7}\right) \\
p_{\text {value }}=1-\Phi\left(Z \leq \frac{0.12-0.1}{\sqrt{1.68 \times 10^{-7}}}\right)<0.01(S 7)
\end{gathered}
$$

So, we may reject $\mathrm{H}_{0}$ at $\alpha=0.01$.

In total, PaDEL-DNN showed significantly better predictions for the "E" and "B" terms. For the "S" and "A" terms, we accept the $\mathrm{H}_{0}$. Thus, PaDEL and QC-MLR showed comparable predictions for these two terms.

S1.5 Comparison with MLFER: The PaDEL-DNN was also compared with MLFER (a built-in method in PaDEL package based on group-contribution method). As shown in Fig. S4, the general trend is that the PaDEL-DNN models outperformed the MLFER in predicting the four solute descriptors. Based on the description of the PaDEL package, the built-in method for MLFER was based on a group-contribution method, ${ }^{2,7}$ which could be the reason for the worse predictions by MLFER. The group-contribution method mostly focuses on discrete local molecular information with insufficient attention to global molecular characteristics so estimates by this approach are not always satisfactory. 7,8

S1.6 Robustness and generalization: Robustness is generally defined as "the ability of a system to resist change without adapting its initial stable configuration". ${ }^{9}$ In other words, how slight changes in the inputs or the configuration of models affect the prediction performance is the criteria for evaluating the model robustness; the lower the variance in the model performance, the higher the robustness of the models. In this study, the 5-fold cross-validation that was repeated multiple times on the training datasets could be approximately treated as a test for the robustness of the models. In each round of the cross-validation, the training data were divided into 5 sub-parts, among which 
four parts were used as the training dataset and the other part was used as the validation dataset. When the training dataset was randomly divided into 5 sub-parts, this could be treated as a slight change in the input data. When the cross-validation was repeated multiple times, if the proposed model is robust, the prediction error should be comparable for each cross-validation. Otherwise, the proposed model is not robust. To compare the robustness of different models, we compared different models on the same training datasets through cross-validation. Robust models generally show low variances in the RMSE and $R^{2}$ values for regression modeling. As shown in Fig. S2, the DNN models in this study are more robust than other models.

Another term commonly associated with robustness is called generalization, which describes the model's ability to make good predictions for new test cases. A model with good generalization ability should achieve comparable predicting performance on both the training and the test datasets. Because of the highly consistent performance between the training and test datasets (Fig. 2), the PaDEL-DNN models in this study showed promising generalization ability.

\section{Text S2. Three dimension-reduction methods}

Three different methods were employed to reduce the input dimension-reduce the number of input features. The total number of features was reduced to around 300, 200, and 150 by these three methods, separately.

S2.1 First method: The first method used the correlation coefficients (Eq. S8) between every input feature and one of the outputs (E, S, A, and B) to drop less important features.

$$
\rho_{X, Y}=\frac{\operatorname{COV}(X, Y)}{\sigma_{X} \cdot \sigma_{Y}}>s
$$

where $\operatorname{COV}(X, Y), \sigma_{X}, \sigma_{Y}, \rho_{X, Y}$, and $s$ represent the covariance between input feature $X$ and output $Y$, the variance of input feature $X$, the variance of output $Y$, the correlation coefficient between $X$ and $Y$, and the threshold value, respectively.

In this method, a $s$ value ranging from 0.6-0.8 was used as the threshold for the $\rho_{X, Y}$ to reduce the input dimension for each solute descriptor. We reduced the input dimension to different degrees (more features were dropped as the $s$ value varied from 0.8 to 0.6 ) and examined the 5 -fold crossvalidation results on the training datasets. The best dimension-reduced model was reported in the main text. $\mathrm{s}>0.8$ or $<0.6$ were also tried during the dimension reduction; however, the $s$ values within these two ranges either dropped too many or too few features and thus were not further 
explored during modeling. A similar result was also observed for the second method, as discussed below.

S2.2 Second method: In the second method, the correlation among the input features (Eq. S9) was used to drop highly correlated features.

$$
\rho_{X_{i}, X_{j}}=\frac{\operatorname{COV}\left(X_{i}, X_{j}\right)}{\sigma_{X_{i}} \cdot \sigma_{X_{j}}}(i \neq j)<s
$$

where $\operatorname{COV}\left(X_{i}, X_{j}\right), \sigma_{X_{i}}, \sigma_{X_{j}}, \rho_{X_{i} X_{j}}$, and $s$ represent the covariance between input features $X_{i}$ and $X_{j}$, the variance of input feature $X_{i}$, the variance of input feature $X_{j}$, the correlation coefficient between $X_{i}$ and $X_{j}$, and the threshold value, respectively.

A $s$ value ranging from 0.6-0.9 was used as the threshold for the $\rho_{X_{i} X_{j}}$ to reduce the input dimension for each solute descriptor. We reduced the input dimension to different degrees (more features were dropped with $s$ varied from 0.6 to 0.9 ) and compared the 5-fold cross-validation results on the training datasets. The best dimension-reduced model was reported in the main text.

S2.3 Third method: The last one used the LASSO to minimize the prediction error and maximize the sparsity of inputs (or minimizes the number of input features) by using L1 regularization (Eq. S10). ${ }^{10}$

$$
\text { loss }=\sum\left(y_{i}-x_{i} \beta\right)^{2}+\lambda \cdot \sum|\beta|
$$

where $y_{i}, x_{i}, \beta$, and $\lambda$ represent the real output, input features, regression coefficient matrix, and penalty coefficient, respectively. The first part of the loss function is the sum of squares, the minimization of which would achieve good predictions of LASSO models. The second part is the L1 penalty, in which the tunable parameter $\lambda$ controls the strength of the penalty. The larger the $\lambda$ value, the higher the penalty. When $\lambda$ equals 0 , it means that there is no penalty and no elements in the coefficient matrix $\beta$ will be set as zero. On the contrary, all the elements in $\beta$ will be set as zero when $\lambda$ is infinitely large. The training process of LASSO will not only try to minimize the sum of squares but also set as many elements in $\beta$ to zero as possible. The final optimal LASSO model achieves a balance between the prediction accuracy and complexity of the model (number of features used for modeling).

For the final LASSO model, attention is paid to the complexity of the model or more specifically the coefficient matrix $\beta .{ }^{11}$ Some elements in the final $\beta$ matrix will be zero, which means that the corresponding features in the inputs do not contribute to the final predictions and can be removed. 
Using this property, we can screen the input features by examining the $\beta$ matrix of a LASSO model. The first step is to build LASSO models using all available input features (for example, 1319 variables in the PaDEL representation after removing features with missing values) by a series of $\lambda$ values. The 5 -fold cross-validation was used to calculate the prediction errors and to select the best LASSO model. Then, the best LASSO model and the $\lambda$ value were identified based on the smallest 5-fold cross-validation error. Lastly, the LASSO model was retrained using the selected $\lambda$ value without cross-validation. The $\beta$ matrix was then examined to find non-zero elements, and the corresponding input features were selected for the follow-up modeling. Briefly, LASSO crossvalidation was used to find the best model for each descriptor, and only the features with non-zero coefficients in the best model were selected.

\section{Text S3. Chemical datasets and Gaussian process regression (GPR) modeling}

S3.1 Chemical datasets: The $\log K_{\text {storage-lipid/water }}$ dataset has $305+22$ chemicals. The evaluation on the $\log K_{\text {storage-lipid/water }}$ dataset included the 305 chemicals, 51 out of the 305 chemicals, and the additional 22 chemicals. In general, the more complex the chemical structures are, the harder it is to predict their $\log K_{\text {storage-lipid/water }}$. The $\log K_{\text {storage-lipid/water }}$ of the 305 chemicals ranged from -2.66 to 9.88; these chemicals contain a large number of chemical groups, such as alkane, ester, ketone, ether, aldehyde, alcohol, acid, halogenated compounds, and aromatic compounds with substitutions. The 51 chemicals (with higher values for the " $\mathrm{A}$ " term) were further used for comparison because some studies found that the "A" term showed some substantial influences in the pp-LFER of $\log K_{\text {storage-lipid/water }}{ }^{12}$ The additional 22 chemicals cover more complex compounds, each with multiple polar functional groups. A plot (Fig. S5) of 5 randomly selected chemicals from the 305, 51 , and 22 chemical sets is provided below.

The BCF dataset was collected by combining several latest modeling studies, ${ }^{13-16}$ so the current dataset is one of the largest for BCF. Since there was no widely used benchmark dataset for BCF, we collected as many data points as possible. The commonly used freesolve $(639$ chemicals in this study) databases mostly have around 640 organic chemicals (634-643), ${ }^{17-19}$ and there are many more data points available for organic chemicals in non-aqueous solvents (5600). ${ }^{17}$ As for the estimated solubility (ESOL) dataset, the original dataset includes 2874 chemicals; ${ }^{20}$ however, some chemicals are charged at $\mathrm{pH}=7$. Later studies mostly used a dataset with around 1130 chemicals (1128 in this study). ${ }^{19,21}$ For these two datasets, we adopted the most widely used datasets, which makes it convenient to compare the models in this study with existing models. 
One should also realize that the ESOL and Freesolve are not the same as other processes such as

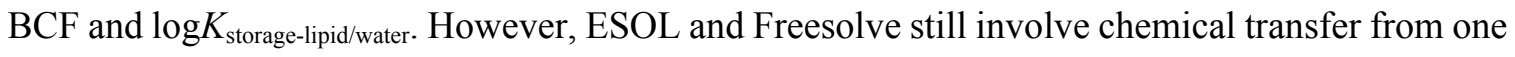
(pure) phase to water despite that the pure phase could be pure liquid or solid. Another very important consideration for selecting ESOL and Freesolve is that the major interactions in these two processes can be largely described by the five solute descriptors. For example, $\log \mathrm{P}$ and molecular weight were reportedly the top two important features for predicting ESOL,$^{20}$ while according to the pp-LFER, $\log P$ can essentially be described by the five solute descriptors and the "V" term can reflect the molecular weight of chemicals.

S3.2 GPR modeling: The Gaussian process regression (GPR) is a simple-to-implement, flexible, and fully probabilistic model, and thus a powerful tool in many areas of application..$^{22}$ GPR models work well on small datasets and can provide uncertainty measurements on predictions. These merits make GPR models suitable for modeling the ESOL, $\operatorname{logBCF}$, or freesolve datasets. The inputs for these three datasets were the five estimated pp-LFER descriptors ( $E, S, A$, and $B$ were predicted by the best PaDEL-DNN models while $V$ was calculated by the well-established method in PaDEL). The GPR modeling was performed in the MATLAB environment. The GPR is a nonparametric regression process; the only tunable parts are the basis function and kernel function. The performance of different combinations of the basis function and kernel function did not show significant differences on these three datasets, but the GPR model using a combination of "constant" basis function and "exponential" kernel function showed slightly better prediction performance and thus was selected for the later modeling. For each of the three processes, the dataset was first randomly split into training and test datasets (8:2). The best model was selected through 5-fold cross-validation on the training datasets and was then evaluated on the reserved test datasets by examining the RMSE and $\mathrm{R}^{2}$ values.

\section{Text S4. The surrogate metric based on $\log P$}

S4.1 Reasons for using the surrogate metric: The surrogate metric based on $\log \mathrm{P}$ rather than traditional ADs was used for three reasons.1) Traditional AD may require the data to follow certain distributions, for example, the leverage method requires a normal distribution. However, values of some descriptors do not follow a normal distribution, such as "A" and "B" (Fig. S1). Thus, some traditional AD methods cannot be directly used. 2) Even if some traditional AD methods can be used, for a new test chemical, we have one AD per solute descriptor. When applying the ADs, some terms such as "E" and "S" may be found within their ADs while the others are not. Hence, it would be a dilemma to decide whether the new test chemical is in the ADs or not. 3) The regular AD is 
based on the model input and lacks model specificity. In other words, there is only one AD per one set of model input regardless of the model output. However, the AD should depend on the model output instead. For example, the values of the "A" term are mostly related to some oxygen/nitrogencontaining function groups while the values of the "E" term may depend on many other functional groups. From the viewpoint of chemical similarity, different chemicals should be deemed similar for "E" versus for "A"; hence, the ADs of "E" and "A" should be different. The post-hoc model analysis indeed showed such results. As cyclohexanamine taken as the example test chemical (Fig. S8), different training chemicals were found to be the most similar chemicals for different solute descriptors (Fig. S9). Because of the above three reasons, this study proposed the surrogate $\log \mathrm{P}$ verification idea. Using the surrogate metric, we do not need to worry about the source data distribution, multiple-AD dilemma, or the model specificity problem. Besides, $\log \mathrm{P}$ is one of the most widely available properties for chemicals.

S4.2 Surrogate metric: The octanol-water partition coefficient $(\log P)$ is one of the most common and useful properties of chemicals. $\log \mathrm{P}$ values are available for tens of thousands of chemicals. Based on the available $\log \mathrm{P}$ values, a good regression relationship between the pp-LFER descriptors and $\log$ P (Eq. 2 in the main text) has been well established. With this relationship, it is possible to determine the overall accuracy of the estimated pp-LFER descriptors for new chemicals by examining the prediction errors for $\log { }^{23}$ For chemicals with newly estimated pp-LFER descriptors, one can employ reported pp-LFER models to predict the $\log$ P for these chemicals. A good prediction for $\log \mathrm{P}$ is likely when the pp-LFER solute descriptors are well estimated; on the contrary, poorly estimated pp-LFER descriptors would likely lead to large prediction errors for $\log$ P. In other words, the accuracy of the estimated pp-LFER descriptors can be evaluated collectively by observing how well they can model $\log \mathrm{P}$, or other chemical transfer-related physical processes, rather than one-by-one evaluation. So, the prediction accuracy for $\log \mathrm{P}$ can be used as a surrogate metric to evaluate the overall accuracy of the estimated pp-LFER descriptors. Besides $\log \mathrm{P}$, other common parameters such as those for chemical transfer between water and 1-propanol or between water and 1-butanol can also serve as surrogate metrics. However, $\log \mathrm{P}$ is the most commonly available property and thus was selected here.

S4.3 The threshold for the surrogate metric: The threshold for the surrogate metric was selected based on the distribution of the prediction errors (Fig. S6) for logP by pp-LFERs, which generally follows a normal distribution $N\left(\mu=0, \sigma^{2}=S D^{2}\right)$. When $3 \times \mathrm{SD}$ is used as the threshold, the probability of a chemical having a prediction error within the range of $(\mu \pm 3 \cdot \mathrm{SD})$ for $\log \mathrm{P}$ is 0.99 . Similarly, the probability is 0.95 or 1 for the threshold of $2 \times \mathrm{SD}$ or $4 \times \mathrm{SD}$, respectively. When we 
apply a threshold on the $\log K_{\text {storage-lipid/water }}$ dataset, the $2 \times \mathrm{SD}, 3 \times \mathrm{SD}$, and $4 \times \mathrm{SD}$ threshold got an RMSE of 0.297 (238 chemicals), 0.3 (274 chemicals), and 0.338 (294 chemicals), respectively. The $2 \times$ SD threshold excluded too many chemicals ( 238 vs. 274 ) but only negligibly improved the prediction (RMSE: 0.297 vs. 0.3 ). The $4 \times$ SD excluded much fewer chemicals but showed almost negligible improvement in the prediction error (RMSE: 0.338 vs. 0.34 ) compared to the predictions without applying the surrogate metric. Overall, the $3 \times \mathrm{SD}$ is a more balanced threshold, which can improve the predictions without excluding too many chemicals.

S4.4 Application of the surrogate metric: The application of the surrogate metric involved two major steps. The first step is to use the PaDEL-DNN models to estimate the pp-LFER descriptors for new chemicals (with $\log \mathrm{P}$ values available). The second step is to use the estimated pp-LFER descriptors to calculate $\log \mathrm{P}$ using Eq. 2 for the chemicals and then to obtain the estimation errors by comparing the reported versus calculated $\log \mathrm{P}$ values. Chemicals with prediction errors smaller than a threshold were classified as "accurate", otherwise as "not accurate". More detailed calculations are shown below.

Step a) Making predictions for the pp-LFER descriptors $\left(M_{L F E R}\right)$ for five example chemicals using the trained PaDEL-DNN models.

$$
M_{\text {LFER }}=\left[\begin{array}{ccccc}
E & S & A & B & V \\
1.76 & 1.65 & 0.01 & 0.15 & 2.22 \\
2.84 & 2.05 & 0.14 & 1.22 & 2.9 \\
1.77 & 1.51 & 0.02 & 0.31 & 2.1 \\
3.00 & 1.81 & 0.04 & 0.57 & 2.06 \\
3.82 & 2.08 & 0.01 & 0.43 & 2.19
\end{array}\right]
$$

Step b) Obtaining the predicted $\log \mathrm{P}$ values $\left(M_{\log P}\right)$ for these five chemicals (the last matrix below) using the estimated solute descriptors (the first matrix) and the reported pp-LFER coefficients (the second matrix) through matrix operation.

$$
M_{\log P}=\left[\begin{array}{llllcc}
1.76 & 1.65 & 0.01 & 0.15 & 2.22 & 1 \\
2.84 & 2.05 & 0.14 & 1.22 & 2.9 & 1 \\
1.77 & 1.51 & 0.02 & 0.31 & 2.1 & 1 \\
3.00 & 1.81 & 0.04 & 0.57 & 2.06 & 1 \\
3.82 & 2.08 & 0.01 & 0.43 & 2.19 & 1
\end{array}\right] \cdot\left[\begin{array}{c}
0.562 \\
-1.054 \\
0.034 \\
-3.46 \\
3.814 \\
0.088
\end{array}\right]=\left[\begin{array}{l}
7.29 \\
6.37 \\
6.43 \\
5.80 \\
6.90
\end{array}\right]
$$

Step c) Calculating the prediction error of $\log P$ for each chemical $\left(E_{\log P}\right)$ by the difference between the predicted (the first matrix) and experimental (the second matrix) $\log P$ values. 


$$
E_{\log P}=\left[\begin{array}{l}
7.29 \\
6.37 \\
6.43 \\
5.80 \\
6.90
\end{array}\right]-\left[\begin{array}{c}
6.91 \\
5.9 \\
6.0 \\
5.5 \\
6.75
\end{array}\right]=\left[\begin{array}{c}
0.38 \\
0.47 \\
0.43 \\
0.3 \\
0.15
\end{array}\right]
$$

Since the prediction errors for these chemicals are all smaller than the threshold value of 0.5 , the overall prediction of the solute descriptors for these chemicals is deemed good ( $M_{\text {goodness }}=$ " $\mathrm{G}$ "). Thus, we can largely trust the overall predictions for these chemicals and can use the predicted solute descriptors to model other chemical transfer processes.

$$
M_{\text {goodness }}=\left[\begin{array}{l}
G \\
G \\
G \\
G \\
G
\end{array}\right]
$$

After adding other chemical identifiers, we got the results shown in Fig. S7 and in the Jupyter notebook.

S4.5 Testing the surrogate metric on the LFER test datasets: To test the above idea, we applied the surrogate metric to the estimated pp-LFER descriptors for the test chemicals from the LFER dataset. The estimation errors for the chemicals classified as "accurate" were smaller than those without applying the surrogate metric. S $(0.208 \pm 0.023$ to $0.165 \pm 0.014)$ and $\mathrm{B}(0.105 \pm 0.011$ to $0.075 \pm 0.005)$ showed the most significant drop in the estimation errors; E $(0.10 \pm 0.01$ to $0.087 \pm 0.008)$ and $A(0.090 \pm 0.06$ to $0.083 \pm 0.01)$ also showed some drop in the estimation errors, suggesting the effectiveness of the surrogate metric.

\section{Text S5. Post hoc interpretation of the obtained PaDEL-DNN models}

S5.1 $K$-nearest neighbor models: The $K$-nearest neighbor models take two steps to make prediction: 1) finding the $K$ (3-50 in this research) most similar chemicals from the training dataset for each test chemical through a suitable similarity metric. The better the used similarity metric is, the lower the prediction errors. (2) Averaging the output values of the selected $K$ nearest training chemicals as the prediction for the test chemical. Among the three $K$-nearest neighbor models, the first and second models selected the nearest neighbors for each test chemical through the standard neighbor algorithm (NearestNeighbors) in the Scikit-learn packages. ${ }^{24}$ The difference between the first and second models is that the first model used the output of the last hidden layer of the trained PaDEL-DNN models as the input of NearestNeighbors, while the second model used the PaDEL 
representation as the input. The third model used the Tanimoto similarity calculated by RDkit to find the nearest neighbors for each test chemical, where training chemicals having the highest similarity with the test chemicals were selected as the nearest neighbors. ${ }^{25}$

In the first model, one group of nearest neighbors per pp-LFER descriptor was selected for each test chemical (four groups in total). In the second and third models, only one group of nearest neighbors were selected per test chemical. As cyclohexanamine (Fig. S8) taken as an example, there are four PaDEL-DNN models for each of the four pp-LFER descriptors. Accordingly, there are four outputs of the last hidden layers. As shown in Fig. S9, five nearest chemicals from the training dataset per pp-LFER descriptor were identified for cyclohexylamine, and different chemicals were selected for different pp-LFER descriptors. In contrast, only one group of 5 nearest chemicals for each test chemical were identified for the four pp-LFER descriptors by the second (Fig. S10) and third (Fig. S11) models.

S5.2 Retrained PaDEL-DNN models with 5 nearest chemicals being excluded: The hyperparameters used for the full PaDEL-DNN models were used here to train the revised PaDELDNN models with 5 nearest chemicals excluded. Briefly, for a given test chemical, five nearest chemicals were removed from the training dataset to obtain a new training dataset per LFER descriptor. The new training dataset was used to train a new PaDEL-DNN model for the corresponding descriptor. The four retrained PaDEL-DNN models were then used to estimate the four LFER descriptors for the above test chemical. The above process was repeated for every chemical in the test dataset. The RMSEs of the predicted LFER descriptors for the test chemicals were then compared between the retrained and the original PaDEL-DNN models.

Additionally, we followed the same training process to retrain the PaDEL-DNN models with 5 random chemicals being excluded. The only difference here is that the 5 chemicals were randomly selected for each test chemical. It should be noted that the number (5) of excluded chemicals was negligible compared to the total number $(>1500)$ of chemicals in the training dataset. When 5 random chemicals were removed, the retrained PaDEL-DNN models (Random) should be able to achieve prediction accuracies comparable to those by the original PaDEL-DNN models.

S5.3 Additional tests for the KNN models: Two additional tests were performed during the KNN modeling to exclude possible overfitting problems given many features in the PaDEL chemical representation. 1) A dimension reduction on the PaDEL chemical representation was performed before the KNN modeling. As shown in Fig. S12, the prediction performance with (PaDEL_Re) and without (PaDEL) the dimension reduction was comparable with only slight differences. These 
results again confirmed no significant over-fitting during the KNN modeling. 2) The test dataset $(\mathrm{N}=396)$ was further randomly split into two datasets to simulate the condition that we have one validation dataset (PaDEL-1H) and one test dataset (PaDEL-2H), each with 198 chemicals.

During modeling, the training and validation datasets are generally used to optimize the model, and the best model will be then evaluated on the test dataset. When overfitting happens, the best model will perform well on the training/validation dataset but not on the test dataset. The KNN modeling process mentioned in the main text was followed independently on these two datasets. For KNN models, the value of $K$ is a very important parameter and can be selected by examining different values (3-50 in this study). The training/validation datasets are mostly used to find the optimal $K$; however, the good performance of the optimal $K$ on the training/validation datasets along is not enough to prove the generalization ability of the model. Thus, a test dataset will be needed to further evaluate the optimal $K$. The model will be satisfactory when the model's performance on the training/validation and test datasets are comparable. In our case, the first small dataset was used as the validation dataset to examine the performance of $\mathrm{KNN}$ models with different $K$ values, with one value likely the optimal one among all the tested $K$ values. After examining a series of $K$ values, rather than only evaluating the KNN model with the optimal $K$ value on the other small dataset (serving as the test dataset), we evaluated all the used $K$ values on the small test dataset. Similar to the comparison of KNN model performance on the training/validation and test datasets using the optimal $K$, we compared the performance of the KNN model on the training/validation and test datasets regarding every $K$ value. If the prediction performance on these two smaller datasets is comparable, it indicates that the KNN modeling in this study does not have serious overfitting issues, otherwise not. As shown in Fig. S13, the RMSEs of the two smaller datasets (PaDEL-1H and PaDEL-2H) were quite comparable, and their performance was comparable to the full test dataset (PaDEL, N=396). The results indicate no significant overfitting during the KNN modeling.

\section{Text S6. Estimating the pp-LFER descriptors for persistent, bio-accumulative, and toxic (PBT) chemicals}

A few recent studies have identified thousands of chemicals as PBT chemicals. ${ }^{26-28}$ However, experimental values for their physical/chemical properties are rarely available. A lack of these key values makes the evaluation of their environmental behaviors or risks almost impossible. The PaDEL-DNN models and the surrogate metric developed in this research can help address part of the problem. 
The literature ${ }^{26-28}$ have listed a considerable number of PBT chemicals. After removing repetitive chemicals and dropping invalid chemical structures or chemicals with inconsistent identifiers (for example, CAS numbers and SMILES are not consistent), a list of 4170 chemicals was compiled. Among the 4170 chemicals, only 150 chemicals have experimental pp-LFER descriptors reported, which suggests the scarcity of experimental parameters for these PBT chemicals. The PaDEL-DNN models were used to estimate the pp-LFER descriptors for the remaining 4020 chemicals, and the surrogate metric was then calculated for these chemicals based on the estimated LFER descriptors. The calculated surrogate metric results (available in the provided excel file 'SourceData.xlxs') showed that satisfactory estimates were obtained for around half of the chemicals. For the remaining PBT chemicals whose pp-LFER descriptor values were not within the surrogate metric threshold (between 0.5-1 log unit), these estimates can still be helpful in modeling research that have slightly lower accuracy requirements.

The prediction performance for the 4020 PBT chemicals also suggests the necessity of applying the surrogate metric. Without it, it is impossible to distinguish good estimates from poor ones, and modeling work using the estimated LFER descriptors would inevitably suffer large uncertainties.
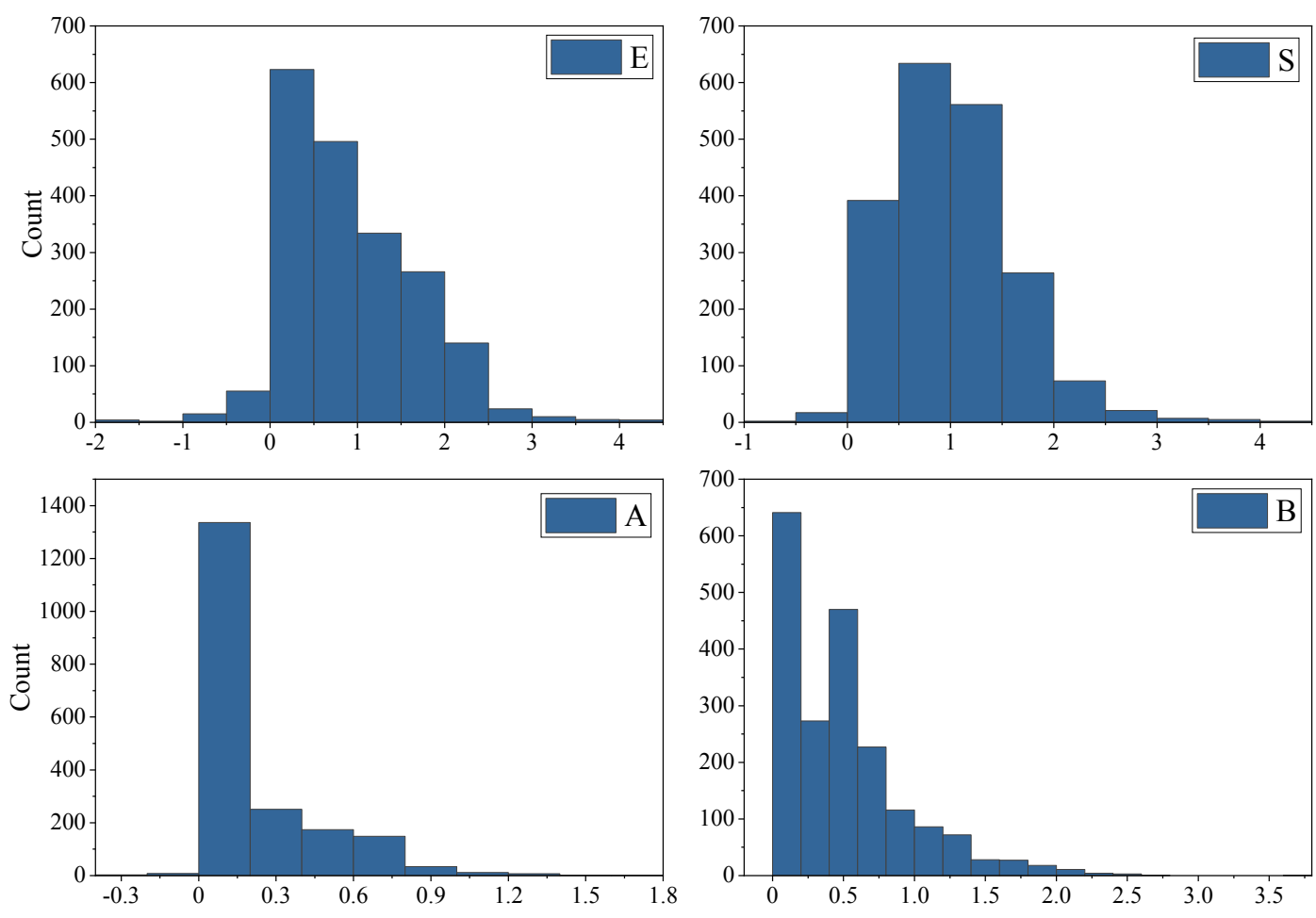

Figure S1. The distribution histograms for the E, S, A, and B values in the LFER dataset 


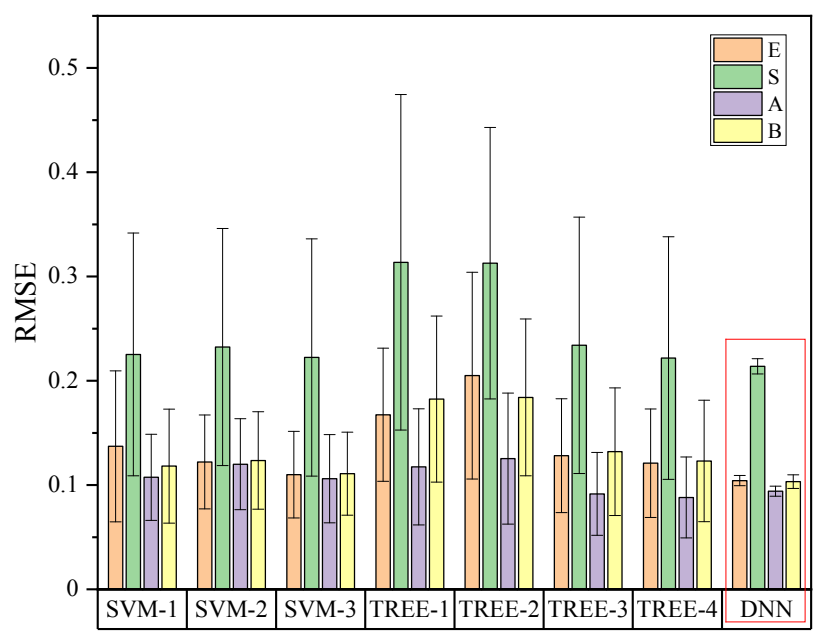

Figure S2. Modeling performance for the cross-validation for different algorithms using the PaDEL chemical representation. SVM-1 to -3 represent SVM with different kernel functions. Tree-1 to -4 indicate models using different tree-based algorithms.
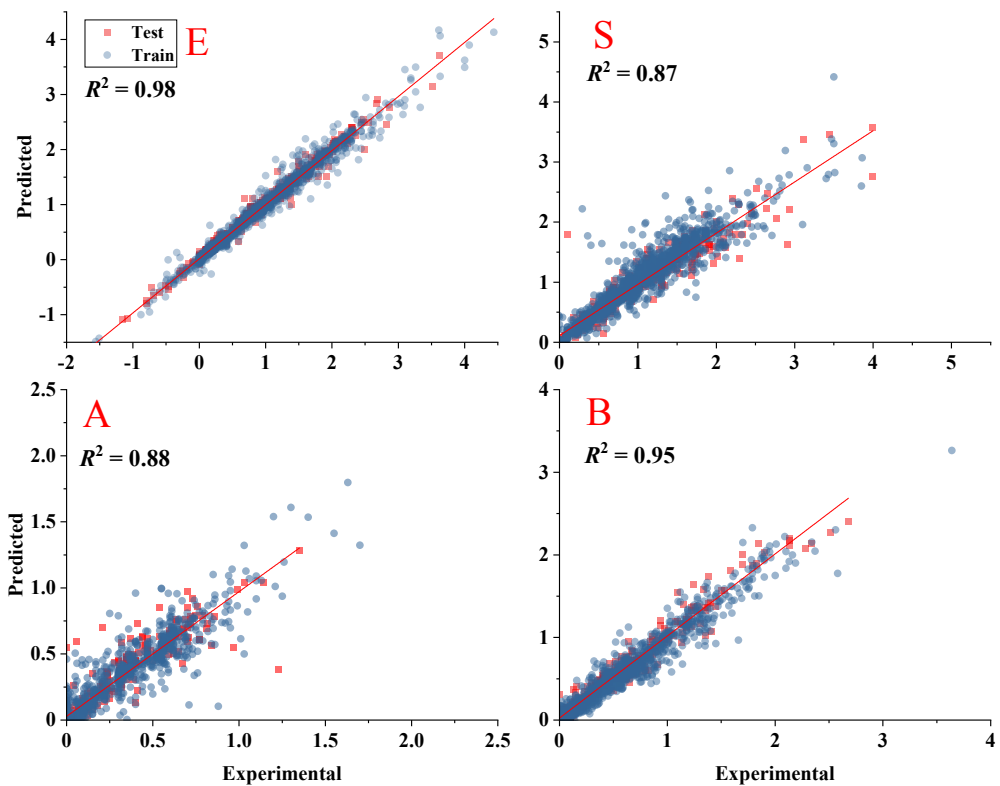

Figure S3. Predicted vs. experimental values for E, S, A, and B by the PaDEL-DNN models. Note, there are more than 1900 data points in each plot. 

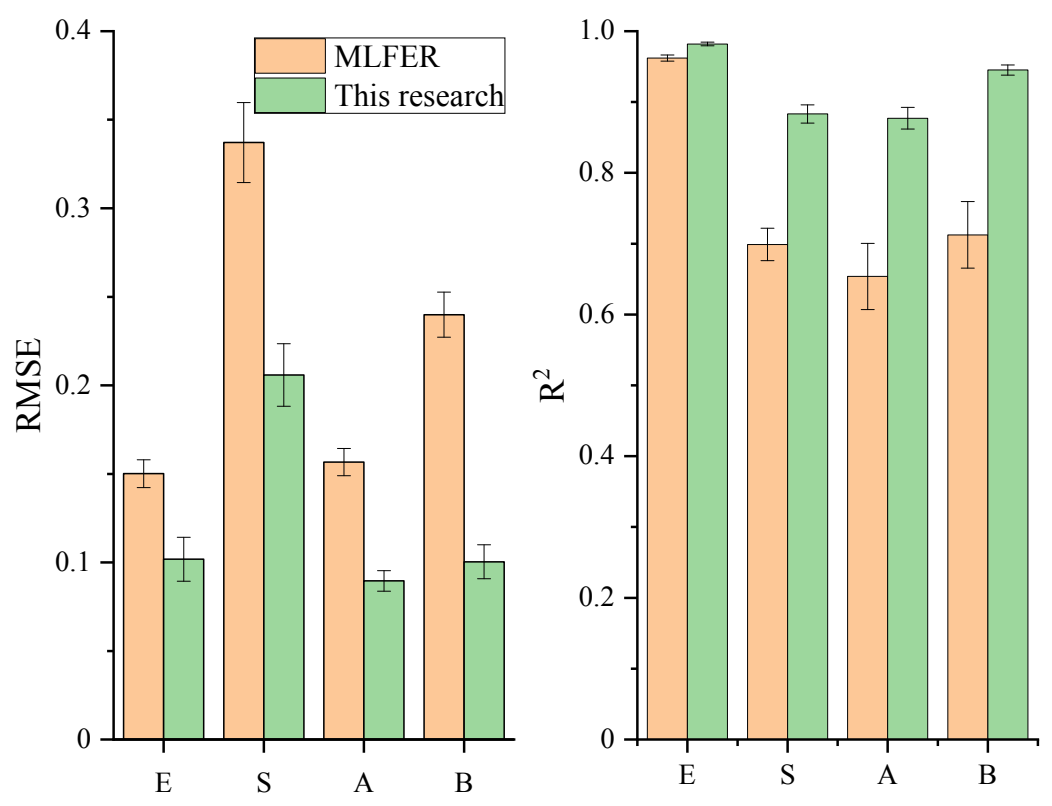

Figure S4. Comparison of the prediction accuracy for the pp-LFER descriptors by the PaDELDNN models (this research) and by the MLFER (the PaDEL package).

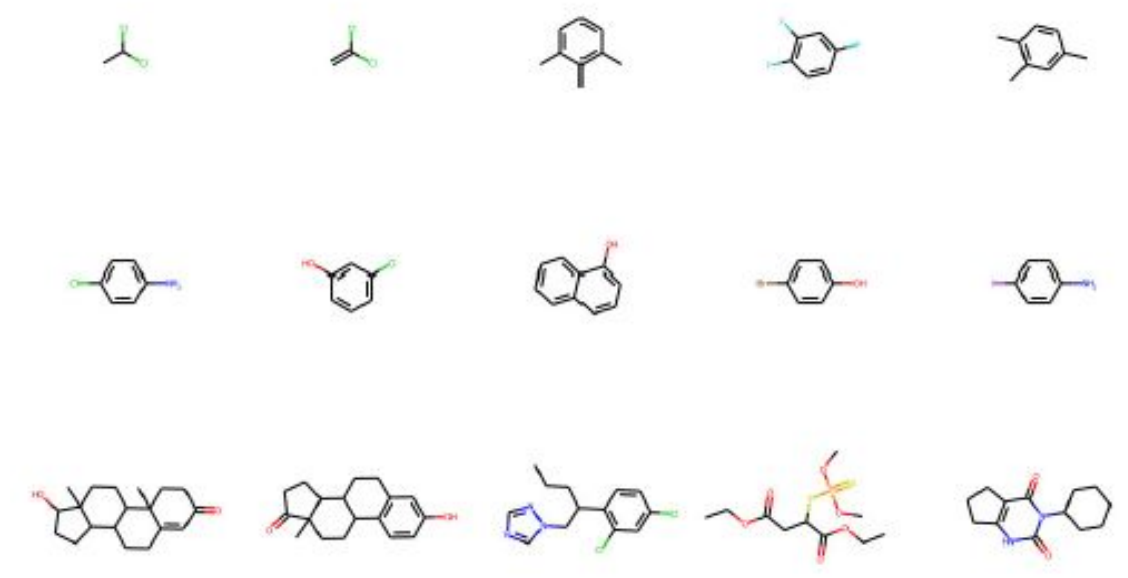

Figure S5. Five chemicals were randomly selected from the 305, 51, and 22 chemical sets, respectively. The chemicals in the first row belong to the 305 -chemical set minus the 51 chemicals; the second row showed 5 chemicals from the 51-chemical dataset; the last row showed 5 chemicals from the 22-chemical set. 


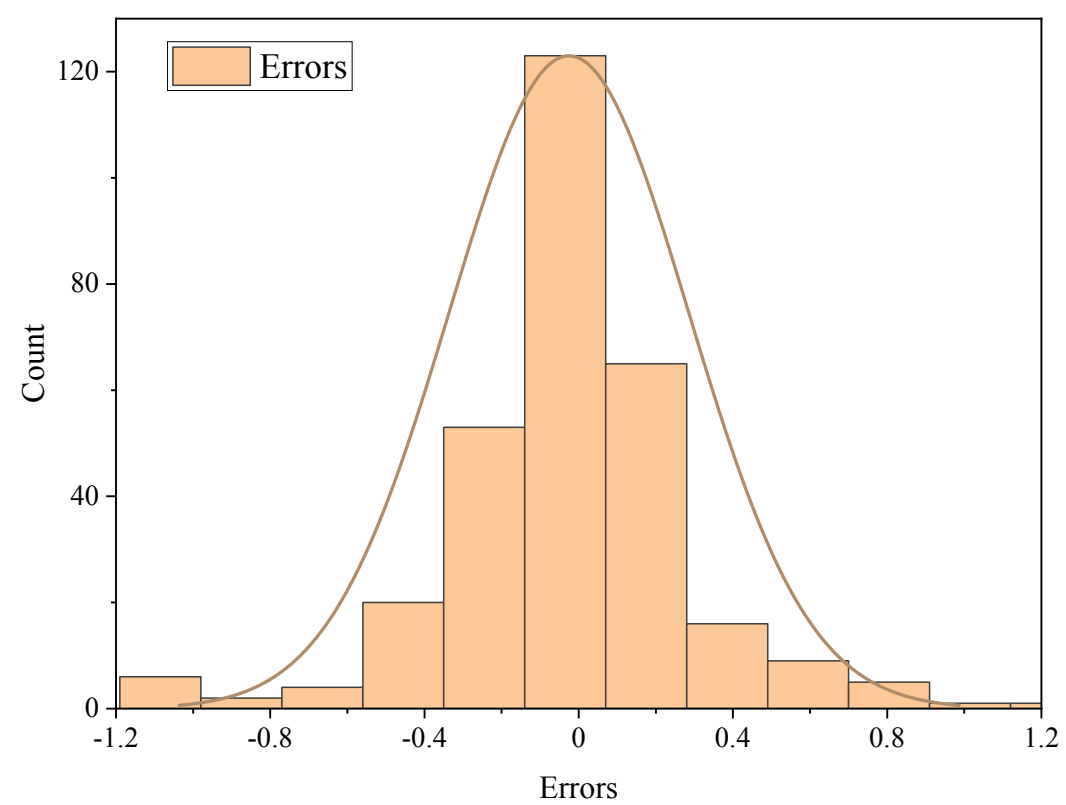

Figure S6. The distribution of the prediction errors of $\log \mathrm{P}$ for the chemicals from the $\log K_{\text {storage- }}$ lipid/water dataset.

[19]: SUILES $=\left[{ }^{2} \mathrm{Clc1} \mathrm{ccc}(\mathrm{cc} 1) \mathrm{C}(\mathrm{c} 2 \mathrm{ccc}(\mathrm{Cl}) \mathrm{cc} 2) \mathrm{c}(\mathrm{Cl})(\mathrm{Cl}) \mathrm{Cl}^{\prime}\right.$

" $\mathrm{CSc} 1 \mathrm{ccc} 2 \mathrm{Se} 3 \operatorname{ccccc} 3 \mathrm{~N}(\mathrm{COC} 4 \mathrm{CcCCN} 4 \mathrm{C}) \mathrm{c} 2 \mathrm{c1} 1^{*}$

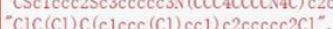

$-\operatorname{col} 1 \mathrm{cc}(\mathrm{C}) \mathrm{c} 2 \mathrm{ne} 3 \mathrm{ccc} 4 \mathrm{ccccc} 4 \mathrm{c} 3 \mathrm{cc} 2 \mathrm{cl}-$.

$-\mathrm{clccc} 2 \mathrm{c}(\mathrm{c} 1) \operatorname{ccc} 3 \cos 4 \mathrm{c}(\mathrm{ccc} 5 \mathrm{ccccc} 45) \mathrm{cc} 23^{*}$

Cc1ecc2 $2 \cos (\operatorname{ccc} 4 \operatorname{ccccc} 34) \operatorname{c5ccc1c25}$,

$" \mathrm{ClC1CC2C}(\mathrm{ClCl}) \mathrm{C} 3(\mathrm{Cl}) \mathrm{C}\left(=\mathrm{C}(\mathrm{Cl}) \mathrm{C}_{2}(\mathrm{Cl}) \mathrm{C} 3(\mathrm{Cl}) \mathrm{Cl}_{1}\right) \mathrm{Cl}^{2}$

" $\mathrm{Cc1c} 2 \operatorname{ccccc} 2 \mathrm{c}(\mathrm{C}) \mathrm{c} 3 \mathrm{c} 4 \mathrm{ccccc} 4 \mathrm{ccc} 13^{\prime}$

[22]: new_prediction $=[]$

models, Scaler, feature name $=$ loading, model 0

for smiles in syivs:

features, V, Inform, cids =generate feature (smiles, feature_name, Scaler)

prediction $=$ making prediction (models, features, $v$, Inform)

new_prediction. append ([1ist (Inform[' synonyms']) [0] [0], title()]+[smiles]+cids+prediction)

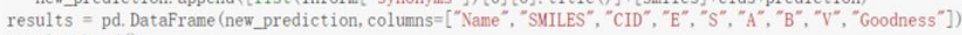

results. head 0

\#results. to_csv (". /results. csv")

\begin{tabular}{|c|c|c|c|c|c|c|c|c|c|}
\hline & Name & SMILES & CID & E & s & A & B & v & Goodness \\
\hline 0 & Clofenotane & $\mathrm{Clc} 1 \mathrm{ccc}(\mathrm{cc} 1) \mathrm{C}(\mathrm{c} 2 \mathrm{ccc}(\mathrm{Cl}) \mathrm{cc} 2) \mathrm{C}(\mathrm{Cl})(\mathrm{Cl}) \mathrm{Cl}$ & 3036 & 1.76 & 1.65 & 0.01 & 0.15 & 2.22 & G \\
\hline 1 & Thioridazine & $\mathrm{CSc} 1 \mathrm{ccc} 2 \mathrm{Sc} 3 \operatorname{ccccc} 3 \mathrm{~N}(\mathrm{CCC} 4 \mathrm{CCCCN} 4 \mathrm{C}) \mathrm{c} 2 \mathrm{c} 1$ & 5452 & 2.84 & 2.05 & 0.14 & 1.22 & 2.90 & G \\
\hline 2 & Mitotane & $\mathrm{ClC}(\mathrm{Cl}) \mathrm{C}(\mathrm{c} 1 \mathrm{ccc}(\mathrm{Cl}) \operatorname{cc} 1) \mathrm{c} 2 \operatorname{ccccc} 2 \mathrm{Cl}$ & 4211 & 1.77 & 1.51 & 0.02 & 0.31 & 2.10 & G \\
\hline 3 & 8,10-Dimethylbenz(A)Acridine & $\mathrm{Cc} 1 \mathrm{cc}(\mathrm{C}) \mathrm{c} 2 \mathrm{nc} 3 \mathrm{ccc} 4 \mathrm{ccccc} 4 \mathrm{c} 3 \mathrm{cc} 2 \mathrm{c} 1$ & 66127 & 3.00 & 1.81 & 0.04 & 0.57 & 2.06 & G \\
\hline 4 & Dibenz[A,H]Anthracene & $\operatorname{c1ccc} 2 c(c 1) \operatorname{ccc} 3 \operatorname{cc} 4 c(\operatorname{ccc} 5 \operatorname{cccc} c 45) \operatorname{cc} 23$ & 5889 & 3.82 & 2.08 & 0.01 & 0.43 & 2.19 & G \\
\hline
\end{tabular}

Figure S7. Demonstration of the application of the surrogate metric 


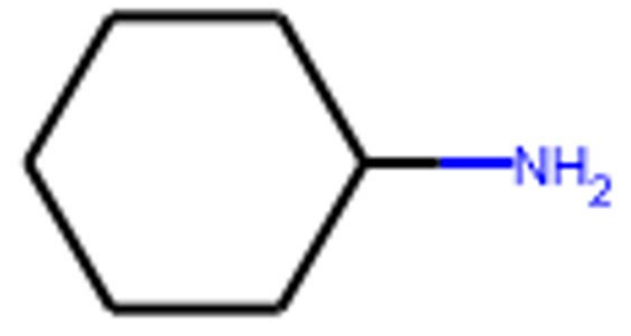

$$
\mathrm{E}=\mathbf{0 . 3 3} \quad \mathrm{S}=\mathbf{0 . 5 6} \quad \mathrm{A}=\mathbf{0 . 1 9} \quad \mathrm{B}=\mathbf{0 . 5 8}
$$

Figure S8. Structure and the pp-LFER descriptor values for cyclohexanamine

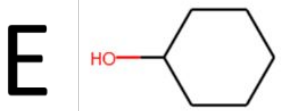

$E=0.46$

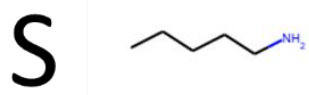

$S=0.35$

A

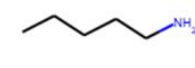

$A=0.16$<smiles>CCCCCCCC</smiles>

$A=0.16$

$A=0.0$

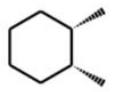

B

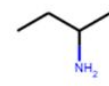

$B=0.63$

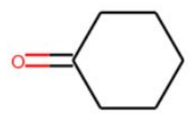

$E=0.4$

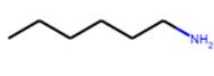

$E=0.35$

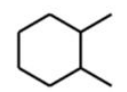

$A=0.0$

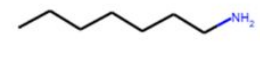

$B=0.61$<smiles>CCCCCCC</smiles>

$B=0.61$
$E=0.39$

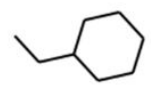

$A=0.0$

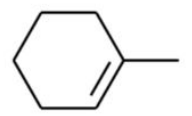

$E=0.39$
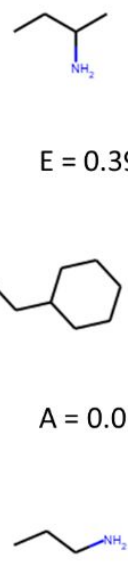

$B=0.61$

Figure S9. Five nearest neighbors for cyclohexanamine based on the outputs of the last hidden layers of the PaDEL-DNN models for E, S, A, and B, respectively. The values under each structure are the corresponding descriptor values. 
E

E<smiles>OC1CCCCC1</smiles><smiles>CCCCC[NH3+]</smiles><smiles>CCC(C)N</smiles>

$E=0.17$

$E=0.46$

$E=0.21$<smiles>CCCCCC</smiles><smiles>CCC(C)N</smiles>

$S=0.32$

$$
S=0.54
$$

$S=0.35$

A<smiles>OC1CCCCC1</smiles>

$A=0.32$

B

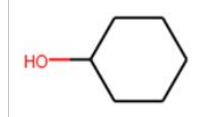

$B=0.57$<smiles>CCCCC[Hg]</smiles><smiles>CCC(C)N</smiles>

$A=0.16$<smiles>CCC(C)N</smiles>

$B=0.63$<smiles>Nc1ccccc1</smiles><smiles>Nc1ccccc1</smiles><smiles>C[C@]1CCCC[C@@]1C</smiles>

$E=0.28$<smiles>C[C@@H]1CCCC[C@H]1C</smiles>

$E=0.24$<smiles>Nc1ccccc1</smiles><smiles>C[C]1CCCC[C@@H]1C</smiles>

$A=0.0$<smiles>Nc1ccccc1</smiles>

$B=0.41$

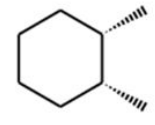

$\mathrm{B}=0.0$

Figure S10. Five nearest neighbors for cyclohexanamine based on the PaDEL representation. The values under each structure are the corresponding descriptor values. 


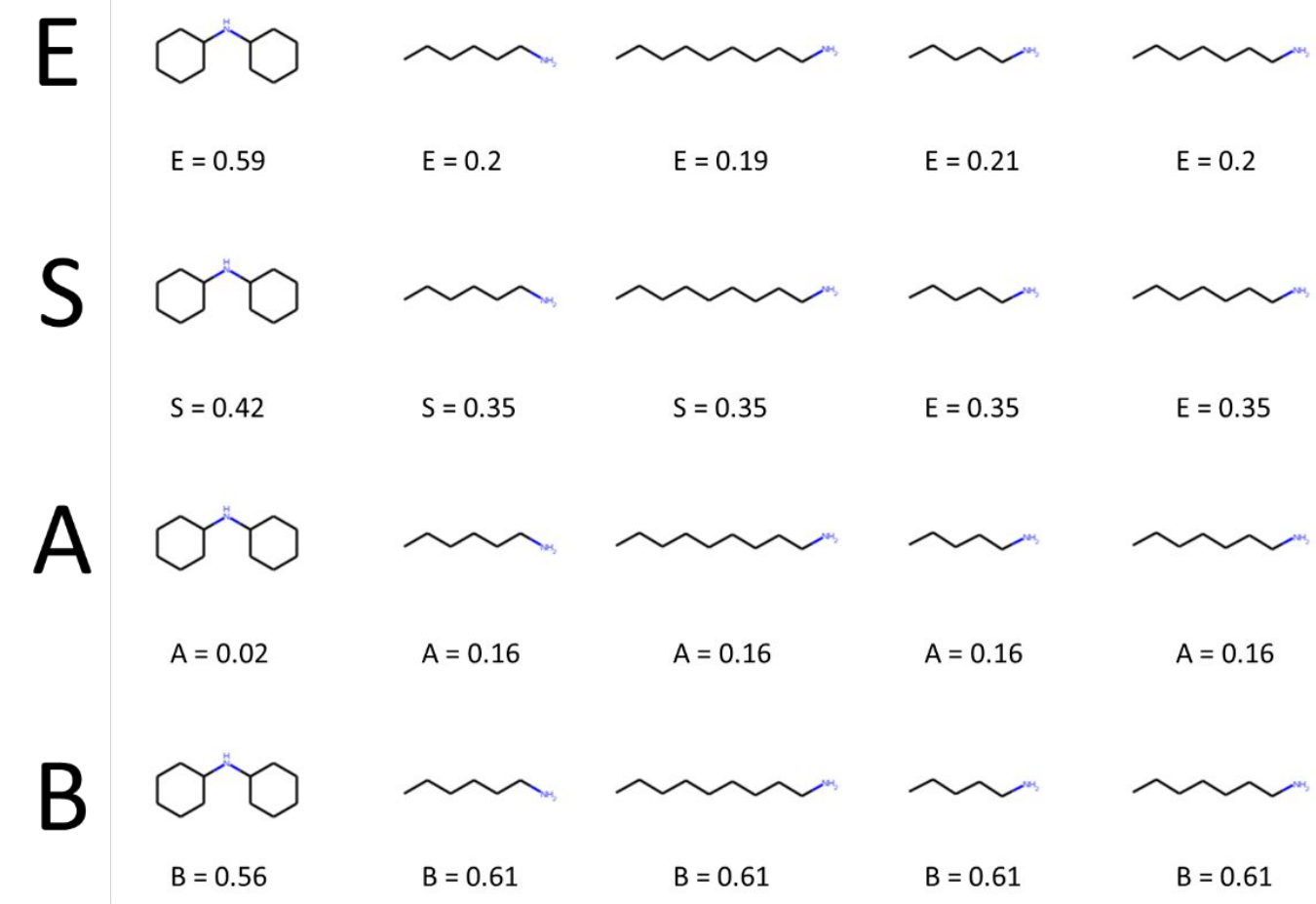

Figure S11. Five nearest neighbors for cyclohexanamine based on the Tanimoto similarity. The values under each structure are the corresponding descriptor values.

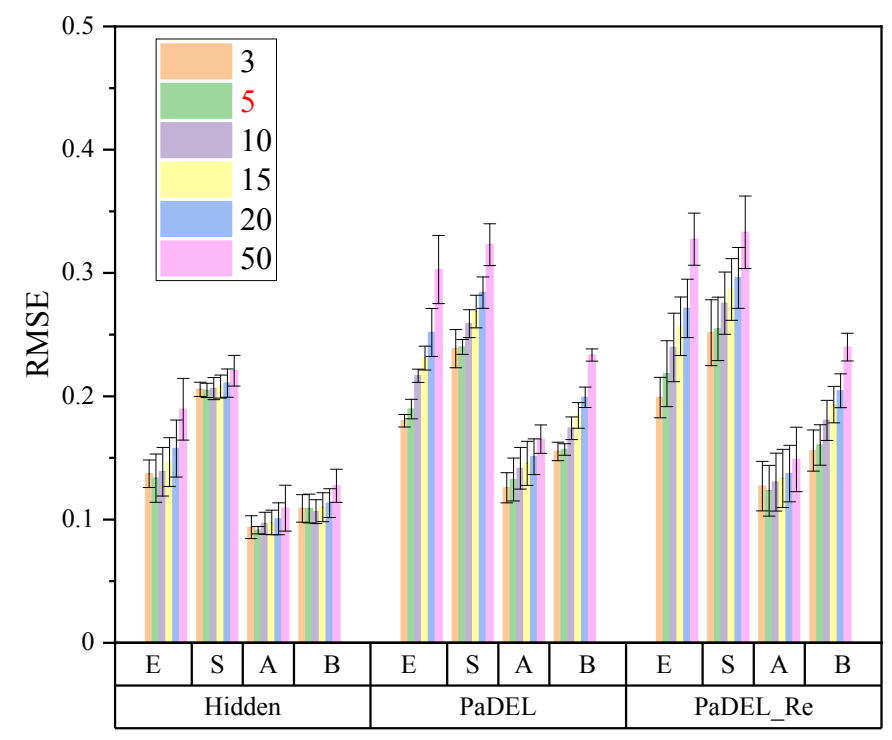

Figure S12. Comparison of the model performance of the KNN models using the PaDEL chemical representation with $\left(\mathrm{PaDEL} \_\mathrm{Re}\right)$ and without (PaDEL) dimension reduction. 


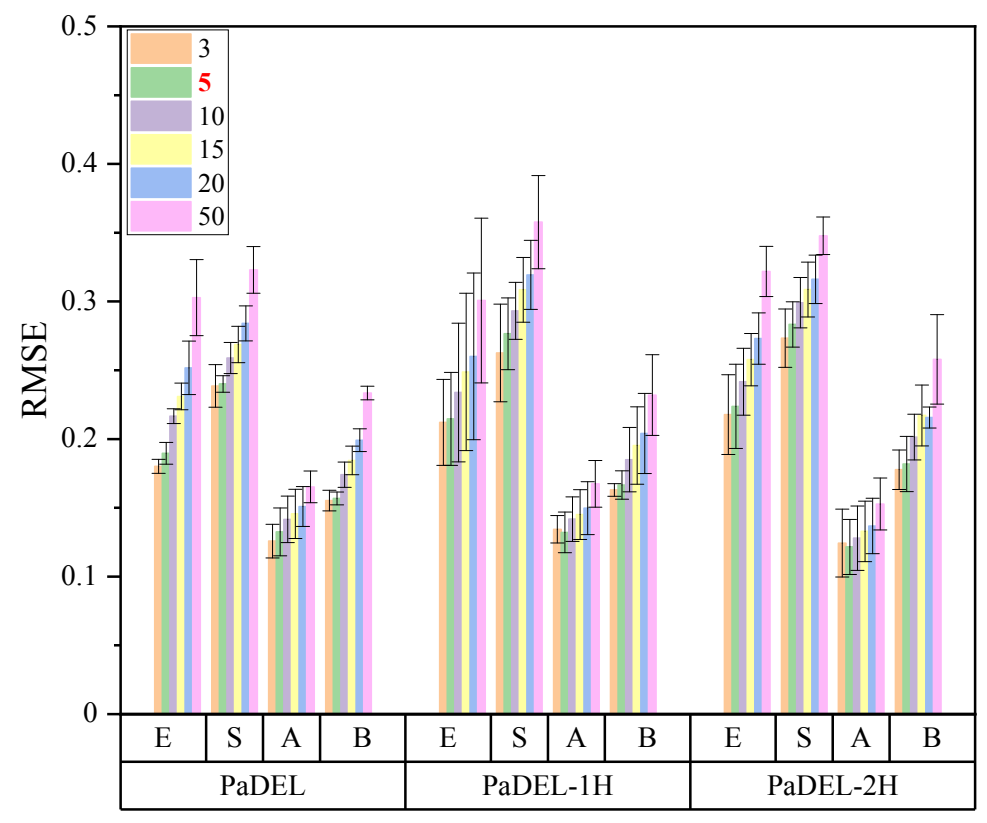

Figure S13. Comparison of the model performance on the full test dataset (PaDEL) and two smaller test datasets (PaDEL-1H and PaDEL-2H).
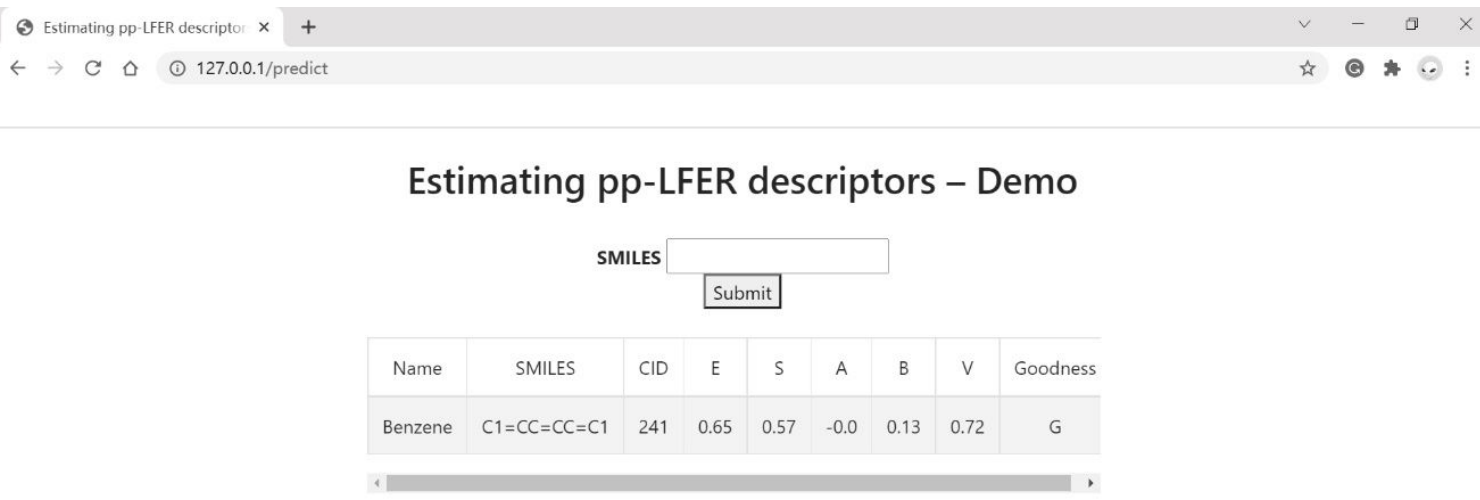

Figure S14. Snapshot of the demo prediction for benzene by the web predictor. "G" for goodness represents the overall prediction is within the threshold of the surrogate metric. If the prediction error falls between 1-2 times the surrogate metric or beyond 2 times the surrogate metric, " $\mathrm{F}$ " or "B" will be displayed instead of "G". CID = PubChem chemical identifier. 
Table S1. Summary of the datasets compiled in this research

\begin{tabular}{cccc}
\hline Name & \# of chemicals & Items & Purpose \\
\hline LFER & 1978 & CID, SMILES, E, S, A, B, V, and $\log P$ & modeling and validation \\
$\log K_{1-w}$ & $305+22$ & CID, SMILES, $\log K_{1-w}$, and $\log \mathrm{P}$ & model validation \\
ESOL & 1128 & CID, SMILES, solubility, and $\log P$ & model validation \\
BCF & 1034 & CID, SMILES, logBCF, and $\log \mathrm{P}$ & model validation \\
freesolve & 642 & CID, SMILES, free energy, and $\log \mathrm{P}$ & model validation \\
PBT & 4020 & CID, SMILES, and $\log P$ & model application \\
\hline
\end{tabular}

Table S2. Comparison of the performance of MF-DNN using molecule fingerprints with different lengths as the chemical representation

\begin{tabular}{cccccc}
\hline $\begin{array}{c}\text { length } \\
\text { (bits) }\end{array}$ & & \multicolumn{2}{c}{ Training } & \multicolumn{2}{c}{ Test } \\
\hline & E & $0.24 \pm 0.01$ & $0.90 \pm 0.01$ & $0.25 \pm 0.04$ & $0.90 \pm 0.03$ \\
512 & $\mathrm{~S}$ & $0.31 \pm 0.01$ & $0.76 \pm 0.01$ & $0.29 \pm 0.05$ & $0.76 \pm 0.07$ \\
& $\mathrm{~A}$ & $0.18 \pm 0.01$ & $0.57 \pm 0.01$ & $0.17 \pm 0.03$ & $0.58 \pm 0.10$ \\
& $\mathrm{~B}$ & $0.20 \pm 0.01$ & $0.80 \pm 0.02$ & $0.21 \pm 0.05$ & $0.78 \pm 0.05$ \\
\hline & $\mathrm{E}$ & $0.23 \pm 0.01$ & $0.91 \pm 0.01$ & $0.23 \pm 0.03$ & $0.91 \pm 0.02$ \\
1024 & $\mathrm{~S}$ & $0.30 \pm 0.01$ & $0.78 \pm 0.01$ & $0.28 \pm 0.04$ & $0.79 \pm 0.04$ \\
& $\mathrm{~A}$ & $0.16 \pm 0.00$ & $0.63 \pm 0.01$ & $0.15 \pm 0.02$ & $0.66 \pm 0.08$ \\
& $\mathrm{~B}$ & $0.19 \pm 0.02$ & $0.84 \pm 0.02$ & $0.19 \pm 0.04$ & $0.84 \pm 0.03$ \\
\hline & $\mathrm{E}$ & $0.22 \pm 0.01$ & $0.91 \pm 0.01$ & $0.22 \pm 0.03$ & $0.92 \pm 0.02$ \\
& $\mathrm{~S}$ & $0.29 \pm 0.01$ & $0.79 \pm 0.01$ & $0.27 \pm 0.04$ & $0.79 \pm 0.06$ \\
& $\mathrm{~A}$ & $0.16 \pm 0.01$ & $0.65 \pm 0.02$ & $0.16 \pm 0.02$ & $0.64 \pm 0.05$ \\
& $\mathrm{~B}$ & $0.18 \pm 0.01$ & $0.85 \pm 0.01$ & $0.18 \pm 0.04$ & $0.85 \pm 0.03$ \\
\hline & $\mathrm{E}$ & $0.22 \pm 0.01$ & $0.92 \pm 0.01$ & $0.21 \pm 0.03$ & $0.92 \pm 0.02$ \\
& $\mathrm{~S}$ & $0.29 \pm 0.01$ & $0.79 \pm 0.01$ & $0.28 \pm 0.04$ & $0.77 \pm 0.05$ \\
& $\mathrm{~A}$ & $0.16 \pm 0.01$ & $0.65 \pm 0.02$ & $0.15 \pm 0.02$ & $0.67 \pm 0.07$ \\
& $\mathrm{~B}$ & $0.18 \pm 0.02$ & $0.84 \pm 0.03$ & $0.17 \pm 0.03$ & $0.85 \pm 0.02$ \\
\hline & $\mathrm{E}$ & $0.22 \pm 0.01$ & $0.92 \pm 0.01$ & $0.22 \pm 0.03$ & $0.92 \pm 0.02$ \\
& $\mathrm{~S}$ & $0.29 \pm 0.01$ & $0.79 \pm 0.01$ & $0.27 \pm 0.04$ & $0.79 \pm 0.05$ \\
& $\mathrm{~A}$ & $0.15 \pm 0.00$ & $0.68 \pm 0.01$ & $0.15 \pm 0.02$ & $0.68 \pm 0.07$ \\
& $\mathrm{~B}$ & $0.18 \pm 0.01$ & $0.85 \pm 0.02$ & $0.18 \pm 0.04$ & $0.85 \pm 0.04$ \\
\hline
\end{tabular}


Table S3 Features included in the RDKit and PaDEL molecular representations used in this research. More detailed information about each term can be found in the literature. ${ }^{1,2}$ Note: each feature may include more than one value. For example, "Atom count" includes counts for total, heavy, nitrogen, carbon atom, etc.

\begin{tabular}{|c|c|}
\hline RDKit & PaDEL \\
\hline Asphericity & Acidic group count \\
\hline AUTOCORR3D & ALOGP \\
\hline BalabanJ & APol \\
\hline BCUT2D & Aromatic atoms count \\
\hline BertzCT & Aromatic bonds count \\
\hline CalcAUTOCORR2D & Atom count \\
\hline CalcChi0n/0v/1n/1v/2n/2v/3v/4n/4v & Autocorrelation \\
\hline CrippenDescriptors & Barysz matrix \\
\hline Eccentricity & Basic group count \\
\hline ExactMolWt & BCUT \\
\hline FpDensityMorgan1 & Bond count \\
\hline FractionCSP3 & BPol \\
\hline GETAWAY & Burden modified eigenvalues \\
\hline HallKierAlpha & Carbon types \\
\hline HeavyAtomCount & Chi chain \\
\hline InertialShapeFactor & Chi cluster \\
\hline Kappa1, 2, 3 & Chi path cluster \\
\hline LabuteASA & Chi path \\
\hline MolLogP & Constitutional \\
\hline MolMR & Crippen $\log \mathrm{P}$ and $\mathrm{MR}$ \\
\hline MORSE & Detour matrix \\
\hline NHOHCount & Eccentric connectivity index \\
\hline NOCount & Atom type electrotopological state \\
\hline NPR1/2 & Extended topochemical atom \\
\hline NumAliphaticCarbocycles & FMFDescriptor \\
\hline NumAliphaticHeterocycles & Fragment complexity \\
\hline NumAliphaticRings & Hbond acceptor count \\
\hline NumAmideBonds & Hbond donor count \\
\hline NumAromaticCarbocycles & Hybridization ratio \\
\hline NumAromaticHeterocycles & Information content \\
\hline NumAromaticRings & Kappa shape indices \\
\hline NumAtomStereoCenters & Largest chain \\
\hline NumBridgeheadAtoms & Largest Pi system \\
\hline NumHAcceptors & Longest aliphatic chain \\
\hline NumHBA & Mannhold LogP \\
\hline NumHBD & McGowan volume \\
\hline NumHDonors & Molecular distance edge \\
\hline
\end{tabular}




\author{
NumHeteroatoms \\ NumHeterocycles \\ NumLipinskiHBA \\ NumLipinskiHBD \\ NumRotatableBonds \\ NumSaturatedCarbocycles \\ NumSaturatedCarbocycles \\ NumSaturatedHeterocycles \\ NumSaturatedHeterocycles \\ NumSaturatedRings \\ NumSpiroAtoms \\ NumValenceElectrons \\ $\mathrm{PBF}$ \\ PMI1/2/3 \\ RadiusOfGyration \\ $\mathrm{RDF}$ \\ RingCount \\ SpherocityIndex \\ TPSA \\ WHIM
}

Molecular linear free energy relation

Path counts

Petitjean number

Ring count

Rotatable bonds count

Rule of five

Topological

Topological charge

Topological distance matrix

Topological polar surface area

Van der Waals volume

Vertex adjacency information

Walk counts

Weight

Weighted path

Wiener numbers

$\mathrm{X} \log \mathrm{P}$

Zagreb index

\section{References}

1. Landrum, G., RDKit: A software suite for cheminformatics, computational chemistry, and predictive modeling. In Academic Press: 2013.

2. Yap, C. W., PaDEL-descriptor: An open source software to calculate molecular descriptors and fingerprints. J. Comput. Chem. 2011, 32 (7), 1466-1474.

3. Abadi, M.; Barham, P.; Chen, J.; Chen, Z.; Davis, A.; Dean, J.; Devin, M.; Ghemawat, S.; Irving, G.; Isard, M. In Tensorflow: A system for large-scale machine learning, 12th \{USENIX\} symposium on operating systems design and implementation (\{OSDI\} 16), 2016; 2016; pp 265283.

4. Bergstra, J.; Yamins, D.; Cox, D. D. In Hyperopt: A python library for optimizing the hyperparameters of machine learning algorithms, Proceedings of the 12th Python in science conference, 2013; Citeseer: 2013; p 20.

5. Devore, J. L.; Berk, K. N.; Carlton, M. A., Modern mathematical statistics with applications. Springer: 2012.

6. Liang, Y.; Xiong, R.; Sandler, S. I.; Di Toro, D. M., Quantum Chemically Estimated Abraham Solute Parameters Using Multiple Solvent-Water Partition Coefficients and Molecular Polarizability. Environ. Sci. Technol. 2017, 51 (17), 9887-9898.

7. Platts, J. A.; Butina, D.; Abraham, M. H.; Hersey, A., Estimation of molecular linear free energy relation descriptors using a group contribution approach. Journal of Chemical Information and Computer Sciences. 1999, 39 (5), 835-845. 
8. Brown, T. N., Predicting hexadecane-air equilibrium partition coefficients $(L)$ using a group contribution approach constructed from high quality data. SAR QSAR Environ Res. 2014, 25 (1), 51-71.

9. Wieland, A.; Wallenburg, C. M., Dealing with supply chain risks: Linking risk management practices and strategies to performance. International journal of physical distribution \& logistics management. 2012.

10. Tibshirani, R., Regression shrinkage and selection via the lasso. Journal of the Royal Statistical Society: Series B (Methodological). 1996, 58 (1), 267-288.

11. Brunton, S. L.; Proctor, J. L.; Kutz, J. N., Discovering governing equations from data by sparse identification of nonlinear dynamical systems. Proceedings of the National Academy of Sciences. 2016, 113 (15), 3932-3937.

12. Geisler, A.; Endo, S.; Goss, K.-U., Partitioning of organic chemicals to storage lipids: elucidating the dependence on fatty acid composition and temperature. Environ. Sci. Technol. 2012, 46 (17), 9519-9524.

13. Petoumenou, M. I.; Pizzo, F.; Cester, J.; Fernandez, A.; Benfenati, E., Comparison between bioconcentration factor (BCF) data provided by industry to the European Chemicals Agency (ECHA) and data derived from QSAR models. Environ. Res. 2015, 142, 529-34.

14. Grisoni, F.; Consonni, V.; Villa, S.; Vighi, M.; Todeschini, R., QSAR models for bioconcentration: is the increase in the complexity justified by more accurate predictions? Chemosphere. 2015, 127, 171-9.

15. Grisoni, F.; Consonni, V.; Vighi, M., Detecting the bioaccumulation patterns of chemicals through data-driven approaches. Chemosphere. 2018, 208, 273-284.

16. Grisoni, F.; Consonni, V.; Vighi, M.; Villa, S.; Todeschini, R., Investigating the mechanisms of bioconcentration through QSAR classification trees. Environ Int. 2016, 88, 198-205.

17. Lim, H.; Jung, Y., MLSolvA: solvation free energy prediction from pairwise atomistic interactions by machine learning. Journal of Cheminformatics. 2021, 13 (1), 56.

18. Mobley, D. L.; Guthrie, J. P., FreeSolv: a database of experimental and calculated hydration free energies, with input files. J. Comput. Aided Mol. Des. 2014, 28 (7), 711-20.

19. Wu, Z.; Ramsundar, B.; Feinberg, E. N.; Gomes, J.; Geniesse, C.; Pappu, A. S.; Leswing, K.; Pande, V., MoleculeNet: a benchmark for molecular machine learning. Chem. Sci. 2018, 9 (2), 513-530.

20. Delaney, J. S., ESOL: Estimating Aqueous Solubility Directly from Molecular Structure. Journal of Chemical Information and Computer Sciences. 2004, 44 (3), 1000-1005.

21. Duvenaud, D.; Maclaurin, D.; Aguilera-Iparraguirre, J.; Gómez-Bombarelli, R.; Hirzel, T.; Aspuru-Guzik, A.; Adams, R. P., Convolutional networks on graphs for learning molecular fingerprints. arXiv preprint arXiv:1509.09292. 2015.

22. Quiñonero-Candela, J.; Rasmussen, C. E., A unifying view of sparse approximate Gaussian process regression. Journal of Machine Learning Research. 2005, 6 (12), 1939-1959. 23. Acree, W. E.; Bowen, K. R.; Horton, M. Y.; Abraham, M. H., Computation of Abraham model solute descriptors for 3-methyl-4-nitrobenzoic acid from measured solubility data. Phys. Chem. Liq. 2016, 55 (4), 482-491.

24. Pedregosa, F.; Varoquaux, G.; Gramfort, A.; Michel, V.; Thirion, B.; Grisel, O.; Blondel, M.; Prettenhofer, P.; Weiss, R.; Dubourg, V., Scikit-learn: Machine learning in Python. the Journal of machine Learning research. 2011, 12, 2825-2830.

25. Bajusz, D.; Rácz, A.; Héberger, K., Why is Tanimoto index an appropriate choice for fingerprint-based similarity calculations? Journal of cheminformatics. 2015, 7 (1), 1-13. 
26. Sun, X.; Zhang, X.; Muir, D. C.; Zeng, E. Y., Identification of Potential PBT/POP-Like Chemicals by a Deep Learning Approach Based on 2D Structural Features. Environ. Sci. Technol. 2020, 54 (13), 8221-8231.

27. Zhang, X.; Sun, X.; Jiang, R.; Zeng, E. Y.; Sunderland, E. M.; Muir, D. C., Screening new persistent and bioaccumulative organics in China's inventory of industrial chemicals. Environ. Sci. Technol. 2020, 54 (12), 7398-7408.

28. Strempel, S.; Scheringer, M.; Ng, C. A.; Hungerbühler, K., Screening for PBT chemicals among the "existing" and "new" chemicals of the EU. Environ. Sci. Technol. 2012, 46 (11), 56805687. 